\title{
Poisson Statistics for the Largest Eigenvalues in Random Matrix Ensembles
}

\author{
Alexander Soshnikov \\ University of California at Davis \\ Department of Mathematics \\ Davis, CA 95616, USA \\ soshniko@math.ucdavis.edu
}

\section{Introduction}

The two archetypal ensembles of random matrices are Wigner real symmetric (Hermitian) random matrices and Wishart sample covariance real (complex) random matrices. In this paper we study the statistical properties of the largest eigenvalues of such matrices in the case when the second moments of matrix entries are infinite. In the first two subsections we consider Wigner ensemble of random matrices and its generalization - band random matrices.

\subsection{Wigner Random Matrices}

A real symmetric Wigner random matrix is defined as a square symmetric $n \times n$ matrix with i.i.d. entries up from the diagonal

$A=\left(a_{j k}\right), a_{j k}=a_{k j}, 1 \leq j \leq k \leq n,\left\{a_{j k}\right\}_{j<k}-$ i.i.d. real random variables.

The diagonal entries $\left\{a_{i i}\right\}, 1 \leq i \leq n$, are usually assumed to be i.i.d. random variables, independent from the off-diagonal entries. A Hermitian Wigner random matrix is defined in a similar way, namely as a square $n \times n$ Hermitian matrix with i.i.d. entries up from the diagonal

$A=\left(a_{j k}\right), a_{j k}=\overline{a_{k j}}, 1 \leq j \leq k \leq n,\left\{a_{j k}\right\}_{j<k}-$ i.i.d. complex random variables.

As in the real symmetric case, it is usually assumed that the diagonal entries $\left\{a_{i i}\right\}, 1 \leq i \leq n$, are i.i.d. (real) random variables independent from the off-diagonal entries.

Ensembles (1) and (2) were introduced in mathematical physics by Eugene Wigner in the 1950s (44, 45, 46]). Wigner viewed these ensembles as a mathematical model to study the statistics of the excited energy levels of heavy nuclei. 
The famous Wigner's semicircle law can be formulated as follows. Let the matrix entries in (11) or (2) be centered random variables with the tail of distribution decaying sufficiently fast, so that all moments exist. Denote by $\lambda_{1} \geq \lambda_{2} \geq \ldots \geq \lambda_{n}$ the eigenvalues of a random matrix $n^{-1 / 2} A$. Then the empirical distribution function of the eigenvalues converges, as $n \rightarrow \infty$, to a non-random limit

$$
\frac{1}{n} \#\left(\lambda_{i} \leq x, \quad 1 \leq i \leq n\right) \rightarrow F(x)=\int_{-\infty}^{x} f(t) d t,
$$

where the density of the semicircle law is given by $f(t)=\frac{1}{\pi \sigma^{2}} \sqrt{2 \sigma^{2}-x^{2}}$, for $t \in[-\sqrt{2} \sigma, \sqrt{2} \sigma]$, and $\sigma^{2}$ is the second moment of matrix entries.

This result was subsequently strengthened by many mathematicians (see e.g. 1], 32], 12]). In its general form (due to Pastur and Girko), the theorem holds if the matrix entries of $A$ satisfy the Lindeberg-Feller condition: $\frac{1}{n^{2}} \sum_{1 \leq i \leq j \leq n} \int_{|x|>\tau \sqrt{n}} x^{2} d F_{i j}(x) \rightarrow 0$, where $F_{i j}(x)$ is the distribution function of $a_{i j}^{(n)}$.

From the analytical point of view, the simplest examples of Wigner random matrices are given by the so-called Gaussian Orthogonal and Unitary Ensembles (GOE and GUE for short). The GUE is defined as the ensemble of $n \times n$ Hermitian matrices with the Gaussian entries $\Re a_{j k} \sim N(0,1 / 2), \Im a_{j k} \sim$ $N(0,1 / 2), \quad 1 \leq j<k \leq n ; \quad a_{i i} \sim N(0,1), \quad 1 \leq i \leq n$ (see (27], chapter 6). The joint distribution of the matrix entries has the form

$$
P(d A)=\text { const }_{n} \exp \left(-\frac{1}{2} \operatorname{Tr}\left(A^{2}\right)\right) d A,
$$

where $d A=\prod_{j<k} d \Re a_{i j} d \Im a_{j k} \prod_{i=1}^{n} d a_{i i}$ is the Lebesgue measure on the space of $n$-dimensional Hermitian matrices. The joint distribution of the eigenvalues is given by its density

$$
p_{n}\left(x_{1}, \ldots, x_{n}\right)=Z_{n}^{-1} \prod_{1 \leq i<j \leq n}\left(x_{i}-x_{j}\right)^{2} \exp \left(-\frac{1}{2} \sum_{i} x_{i}^{2}\right) .
$$

The normalization constants in (4) and (5) are known. What is more, one can calculate explicitely the $k$-point correlation functions (see [27, chapter 6 ). This allows one to study the local distribution of the eigenvalues, both in the bulk of ths spectrum and at its edges in great detail. In particular, a celebrated result of Tracy and Widom (see [40]) states that

$$
\lim _{n \rightarrow \infty} \operatorname{Pr}\left(\lambda_{\max } \leq 2 \sqrt{n}+\frac{s}{n^{1 / 6}}\right)=F_{2}(s)=\exp \left(-\int_{s}^{+\infty}(x-s) q^{2}(x) d x\right),
$$

where $q(x)$ is the solution of the Painléve II differential equation

$$
q^{\prime \prime}(x)=x q(x)+2 q^{3}(x)
$$


with the asymptotics at infinity $q(x) \sim A i(x)$ at $x=+\infty$.

The limiting $k$-point correlation function at the edge of spectrum is given by the formula

$$
\rho_{k}\left(x_{1}, \ldots, x_{k}\right)=\operatorname{det}\left(K\left(x_{i}, x_{j}\right)\right)_{1 \leq i, j \leq k},
$$

where

$$
K(x, y)=K_{\text {Airy }}(x, y)=\frac{A i(x) A i^{\prime}(y)-A i^{\prime}(x) A i(y)}{x-y}
$$

is a so-called Airy kernel. We refer the reader to [40] and [13] for the details. We recall that the $k$-point correlation function is defined in such a way that for any disjoint subintervals of the real line $I_{1}, I_{2}, \ldots, I_{k}$, one has

$$
E \prod_{i=1}^{k} \#\left(I_{i}\right)=\int_{I_{1}} \ldots \int_{I_{k}} \rho_{k}\left(x_{1}, \ldots, x_{k}\right) d x_{1} \ldots d x_{k}
$$

where $\#(I)$ denotes the number of the eigenvalues in $I$. A probabilistic interpretation of the above formula is that $\rho_{k}\left(x_{1}, \ldots, x_{k}\right) d x_{1} \ldots d x_{k}$ is the probability to find an eigenvalue in each of the $k$ infinitesimal intervals $\left[x_{i}, x_{i}+\right.$ $\left.d x_{i}\right], i=1, \ldots, k$.

The Gaussian Orthogonal Ensemble (GOE) is defined as the ensemble of $n \times n$ Wigner real symmetric random matrices with the Gaussian entries. More precisely, we assume that $a_{i j}, \quad 1 \leq i \leq j \leq n$, are independent Gaussian $N\left(0,1+\delta_{i j}\right)$ random variables (see e.g. [27, chapter 7$)$. The joint distribution of the matrix entries has the form

$$
P(d A)=c_{n} \exp \left(-\frac{1}{4} \operatorname{Tr}\left(A^{2}\right)\right) d A,
$$

where $d A=\prod_{i \leq j} d a_{i j}$ is the Lebesgue measure on the space of $n$-dimensional real symmetric matrices. The distribution (9) induces the joint distribution of the eigenvalues of the GOE matrix, given by its density

$$
p_{n}\left(x_{1}, \ldots, x_{n}\right)=Z_{n}^{-1} \prod_{1 \leq i<j \leq n}\left|x_{i}-x_{j}\right| \exp \left(-\frac{1}{4} \sum_{i} x_{i}^{2}\right) .
$$

The limiting distribution of the (normalized) largest eigenvalue of a GOE matrix was calculated by Tracy and Widom in (41]).

$$
\lim _{n \rightarrow \infty} \operatorname{Pr}\left(\lambda_{\max } \leq 2 \sqrt{n}+\frac{s}{n^{1 / 6}}\right)=F_{1}(s)=\exp \left(-\frac{1}{2} \int_{s}^{+\infty} q(x)+(x-s) q^{2}(x) d x\right),
$$

The Tracy-Widom distribution (11) was obtained by studying the asymptotic properties of the $k$-point correlation functions at the edge of the spectrum. The $k$-point correlation function in the GOE ensemble has the pfaffian form. In 
the limit $n \rightarrow \infty$ the $k$-point correlation function at the edge of the spectrum is given by the following formula

$$
\rho_{k}\left(x_{1}, \ldots, x_{k}\right)=\left(\operatorname{det}\left(K\left(x_{i}, x_{j}\right)\right)_{1 \leq i, j \leq k}\right)^{1 / 2},
$$

where $K(x, y)$ is a $2 \times 2$ matrix kernel such that

$$
\begin{aligned}
K_{11}(x, y) & =K_{22}(y, x)=K_{\text {Airy }}(x, y)+\frac{1}{2} A i(x) \int_{-\infty}^{y} A i(t) d t \\
K_{12}(x, y) & =-\frac{1}{2} A i(x) A i(y)-\frac{\partial}{\partial y} K_{\text {Airy }}(x, y) \\
K_{21}(x, y) & =\int_{0}^{+\infty}\left(\int_{x+u}^{+\infty} A i(v) d v\right) A i(x+u) d u-\epsilon(x-y)+\frac{1}{2} \int_{y}^{x} A i(u) d u \\
& +\frac{1}{2} \int_{x}^{+\infty} A i(u) d u \int_{-\infty}^{y} A i(v) d v
\end{aligned}
$$

where $\epsilon(z)=\frac{1}{2} \operatorname{sign}(z)$.

\subsection{Band Random Matrices}

A band random matrix is a generalization of a Wigner random matrix ensemble (11), (2). A real symmetric (aperiodic) band random matrix is defined as a square symmetric $n \times n$ matrix $A=\left(a_{j k}\right)$ such that $a_{i j}=0$ unless $|i-j| \leq d_{n}$, and

$$
\left\{a_{j k}, j \leq k ;|j-k| \leq d_{n}\right\}-\text { i.i.d. real random variables. }
$$

A Hermitian band random matrix is defined in a similar way, namely as a square $n \times n$ Hermitian matrix $A=\left(a_{j k}\right)$, such that $a_{i j}=0$ unless $|i-j| \leq d_{n}$, and

$$
\left\{a_{j k}, j \leq k ;|j-k| \leq d_{n}\right\}-\text { i.i.d. complex random variables. }
$$

If $d_{n}=n-1$, we obtain the Wigner ensemble of random matrices. A matrix is called a periodic band matrix if $|i-j|$ is replaced above by $|i-j|_{1}=$ $\min (|i-j|, n-|i-j|)$. Band random matrices have been studied in the last fifteen years (see for example [6], [7], 30], [17]). In the periodic case, the limiting distribution of the eigenvalues of $d_{n}^{-1} A$ is given by the semi-circle law, provided matrix entries have a finite second moment. In the aperiodic case, the limiting distribution of the eigenvalues is different from the semi-circle law, unless $d_{n} / n \rightarrow 0$ (see e.g. 30]). One of the most interesting problems involving band random matrices is the localization/ delocalization properties of the eigenvalues. It is conjectured in physical literature, that the eigenvalues of band random matrices are localized if $d_{n}=O\left(n^{1 / 2}\right)$. As far as we know, there are no rigorous results yet in this direction. 


\subsection{Sample Covariance Random Matrices}

Sample covariance random matrices have been studied in mathematical statistics for the last seventy-five years. We refer to 31], [43 and [19] for the applications of spectral properties of Wishart random matrices in multivariate statistical analysis.

Let $A$ be a large $m \times n$ real rectangular random matrix with independent identically distributed entries. In applications, one is often interested in the statistical behavior of the singular values of $A$ in the limit $m \rightarrow \infty, n \rightarrow \infty$. This is equivalent to studying the eigenvalues of a positive-definite matrix $M=A^{t} A$ in the limit of large dimensions. Without loss of generality, one can assume that $m \geq n$ (since the spectrum of $A A^{t}$ differs from the spectrum of $A^{t} A$ only by a zero eigenvalue of multiplicity $m-n$.

The analogue of the Wigner semicircle law was proved by Marchenko and Pastur (24]). Let $m \rightarrow \infty, n \rightarrow \infty$ in such a way that $m / n \rightarrow \gamma \geq 1$. Assume $E\left|a_{i j}\right|^{2+\epsilon}<+\infty$, where $\epsilon>0$ is an arbitrary positive number. Then the empirical distribution function of the eigenvalues of $\frac{1}{m} A^{t} A$ converges to a non-random limit, known as the Marchenko-Pastur distribution

$$
\frac{1}{n} \#\left(\lambda_{i} \leq x, i=1, \ldots, n\right) \rightarrow G_{\gamma}(x)=\int_{-\infty}^{x} g_{\gamma}(t) d t,
$$

where the spectral density $g(t)$ is supported on the interval $[a, b], a=\sigma^{2}(1-$ $\left.\gamma^{-1 / 2}\right)^{2}, b=\sigma^{2}\left(1+\gamma^{-1 / 2}\right)^{2}, \sigma^{2}=E a_{11}^{2}$, and $g(t)=\frac{1}{2 \pi t \gamma \sigma^{2}} \sqrt{(b-t)(t-a)}, t \in$ $[a, b]$.

The case $a_{i j} \sim N(0,1) \quad 1 \leq i, j \leq n$, is known in the literature as the Wishart (Laguerre) ensemble of real sample covariance matrices. The joint distribution of the eigenvalues of $M$ is defined by its density. Similarly to the Gaussian ensembles of real symmetric and Hermitian matrices discussed in Subsection 1.1, many important statistical quantities in the Wishart ensemble can be calculated explicitely. For example, the joint probability density of the eigenvalues is given by the formula

$$
p_{n}\left(x_{1}, \ldots, x_{n}\right)=Z_{n, m}^{-1} \prod_{1 \leq i<j \leq n}\left|x_{i}-x_{j}\right| \prod_{i=1}^{n} x_{i}^{m-n-1} \exp \left(-x_{i} / 2\right) .
$$

It was shown by Johnstone (21]), that the largest eigenvalue of a Wishart random matrix converges, after a proper rescaling, to the Tracy-Widom distribution $F_{1}$. Namely, let $m \rightarrow \infty, n \rightarrow \infty, m / n \rightarrow \gamma$ and $\mu_{m, n}=$ $\left(n^{1 / 2}+m^{1 / 2}\right)^{2}, \quad \sigma_{m, n}=\left(n^{1 / 2}+m^{1 / 2}\right)\left(n^{-1 / 2}+m^{-1 / 2}\right)^{1 / 3}$. Then

$$
\operatorname{Pr}\left(\lambda_{\max }\left(A^{t} A\right) \leq \mu_{m, n}+s \sigma_{m, n}\right) \rightarrow F_{1}(s)
$$

One can also show (see 37), that the rescaled $k$-point correlation function at the edge of the spectrum converge in the limit to (12).

Finally, we want to remark, that there is a long-standing interest in nuclear physics in the spectral properties of the complex sample covariance matrices 
$A^{*} A$, where the entries of a reactangular matrix $A$ are independent identically distributed complex random variables (see e.g. 46, [5], 16, 42, [2]). We refer the reader to [37 and the references therein for additional information.

\subsection{Universality in Random Matrices}

The universality conjecture in Random Matrix Theory states, loosely speaking, that the local statistical properties of a few eigenvalues in the bulk or at the edge of the spectrum are independent of the distribution of individual matrix entries in the limit of large dimension. The only thing that should matter is, whether the matrix is real symmetric, Hermitian or self-dual quaternion Hermitian.

For Wigner random matrices, the conjecture was rigorously proven at the edge of the spectrum, both for real symmetric and Hermitian case in [36], provided that all moments of matrix entries exist and do not grow faster than the moments of a Gaussian distribution, and the odd moments vanish. In particular, it was shown that the largest eigenvalue, after proper rescaling, converges in distribution to the Tracy-Widom law. In the bulk of the spectrum, the conjecture was proven by Johansson (20]) for Wigner Hermitian matrices, provided the marginal distribution of a matrix entry has a Gaussian component. We refer to 11] and references therein for the universality results in the unitary ensembles of random matrices.

The situation for sample covariance random matrices is quite similar (see papers by Soshnikov [37 and Ben Arous and Péché [3]).

The natural question is how general such results are? What happens if matrix entries have only a finite number of moments? In this article we consider the extreme case when the entries of $A$ do not have a finite second moment. In the next section, we discuss spectral properties of Wigner random matrices and, more generally, band random matrices when marginal distribution of matrix entries has heavy tails. As was shown in [38, the statistics of the largest eigenvalues of such matrices are given by a Poisson inhomogeneous random point process. In Section 3 we discuss a similar result (although in a weaker form) for the largest eigenvalues of sample covariance random matrices with Cauchy entries. Section 4 is devoted to conclusions.

\section{Wigner and Band Random Matrices with Heavy Tails of Marginal Distributions}

In this section we consider ensembles of Wigner real symmetric and Hermitian matrices (1) and (2), and band real symmetric and Hermitian random matrices (16), (2) with the additional condition on the tail of the marginal distribution

$$
G(x)=\operatorname{Pr}\left(\left|a_{j k}\right|>x\right)=\frac{h(x)}{x^{\alpha}},
$$


where $0<\alpha<2$ and $h(x)$ is a slowly varying function at infinity in a sense of Karamata (22, 34). In other words, $h(x)$ is a positive function for all $x>0$, such that $\lim _{x \rightarrow \infty} \frac{h(t x)}{h(x)}=1$ for all $t>0$. The condition (21) means that the distribution of $\left|a_{i j}\right|$ belongs to the domain of the attraction of a stable distribution with the index $\alpha$ (see e.g. [18, Theorem 2.6.1).

Without loss of generality, we restrict our attention to the real symmetric case. The results in the Hermitian case are practically the same. Wigner random matrices (11), (2) with the heavy tails (21), in the special case when limit $\lim _{x \rightarrow \infty} h(x)>0$ exists, were considered on a physical level of rigor by Cizeau and Bouchaud in 8 . They argued, that the typical eigenvalues of $A$ are of the order of $n^{1 / \alpha}$. Cizeau and Bouchaud also suggested a formula for the limiting spectral density of the empirical distribution function of the eigenvalues of $n^{-1 / \alpha} A$. Unlike the Wigner semicircle and Marchenko-Pastur laws, the conjectured limiting spectral density is supported on the whole real line. It was given as

$$
f(x)=L_{\alpha / 2}^{C(x), \beta(x)}(x),
$$

where $L_{\alpha}^{C, \beta}$ is a density of a centered Lévy stable distribution defined through its Fourier transform $\hat{L}(k)$ :

$$
\begin{aligned}
& L_{\alpha}^{C, \beta}=\frac{1}{2 \pi} \int d k \hat{L}(k) e^{i k x}, \\
& \ln \hat{L}(k)=-C|k|^{\alpha}(1+i \beta \operatorname{sgn}(k) \tan (\pi \alpha / 2)),
\end{aligned}
$$

and functions $C(x), \quad \beta(x)$ satisfy a system of integral equations

$$
\begin{aligned}
& C(x)=\int_{-\infty}^{+\infty}|y|^{\frac{\alpha}{2}-2} L_{\alpha / 2}^{C(y), \beta(y)}\left(x-\frac{1}{y}\right) d y, \\
& \beta(x)=\int_{x}^{+\infty} L_{\alpha / 2}^{C(y), \beta(y)}\left(x-\frac{1}{y}\right) d y .
\end{aligned}
$$

We would like to draw the reader's attention to the fact that the density in (22) is not a density of a Lévy stable distribution, since $C(x), \beta(x)$ are functions of $x$. Cizeau and Bouchaud argued, that the density $f(x)$ should decay as $\frac{1}{x^{1+\alpha}}$ at infinity, thus suggesting that the largest eigenvalues of $A$ (in the case $h(x)=$ const) should be of order $n^{\frac{2}{\alpha}}$, and not $n^{\frac{1}{\alpha}}$, which is the order of typical eigenvalues.

Even though originally proven in 38] in the Wigner case, the theorem written below holds in the general case of band random real symmetric (or Hermitian) random matrices (16), (17).

Let $N_{n}$ be the number of independent (i.e. $i \leq j$ ), non-zero matrix entries $a_{i j}$ in $A$. In other words, let $N_{n}=\#\left(1 \leq i \leq j \leq n, \quad|i-j| \leq d_{n}\right)$ in the aperiodic band case, and $N_{n}=\#\left(1 \leq i \leq j \leq n, \quad|i-j|_{1} \leq d_{n}\right)$ in the periodic band case. It is not difficult to see, that $N_{n}=\frac{n(n+1)}{2}$ in the 
Wigner case, $N_{n}=n\left(d_{n}+1\right)$ in the periodic band case, and $N_{n}=n \times$ $\left(d_{n}+1\right)-\frac{d_{n}\left(d_{n}+1\right)}{2}$ in the aperiodic band case. Let us define a normalization constant $b_{n}$ in such a way that

$$
\lim _{n \rightarrow \infty} N_{n} G\left(b_{n} x\right)=\frac{1}{x^{\alpha}}
$$

for all positive $x>0$, where the tail distribution $G$ has been defined in (21). Normalization $b_{n}$ naturally appears (see 23] and Remark 1 below), when one studies the extremal values of a sequence of $N_{n}$ independent identically distributed random variables (21). In particular, one can choose

$$
b_{n}=\inf \left\{t: G(t-0) \geq \frac{1}{N_{n}} \geq G(t+0) .\right.
$$

It follows from (27) and (28), that $N_{n}^{\alpha-\delta} \ll b_{n} \ll N_{n}^{\alpha+\delta}$ for arbitrary small positive $\delta$, and $\frac{N_{n} h\left(b_{n}\right)}{b_{n}^{\alpha}} \rightarrow 1$ as $n \rightarrow \infty$.

Theorem 1 claims that the largest eigenvalues of $A$ have Poisson statistics in the limit $n \rightarrow \infty$.

Theorem 1. Let $A$ be a band real symmetric (16) or Hermitian (17) random matrix with a heavy tail of the distribution of matrix entries (21). Then the random point configuration composed of the positive eigenvalues of $b_{n}^{-1} A$ converges in distribution on the cylinder sets to the inhomogeneous Poisson random point process on $(0,+\infty)$ with the intensity $\rho(x)=\frac{\alpha}{x^{1+\alpha}}$.

In other words, let $0<x_{1}<y_{1}<x_{2}<y_{2}<\ldots x_{k}<y_{k} \leq+\infty$, and $I_{j}=\left(x_{j}, y_{j}\right), j=1, \ldots k$, be disjoint intervals on the positive half-line. Then the counting random variables $\#\left(I_{j}\right)=\#\left(1 \leq i \leq n: \lambda_{i} \in I_{j}\right), \quad j=1, \ldots, k$, are independent in the limit $n \rightarrow \infty$, and have a joint Poisson distribution with the parameters $\mu_{j}=\int_{I_{j}} \rho(x) d x$, i.e.

$$
\lim _{n \rightarrow \infty} \operatorname{Pr}\left(\#\left(I_{j}\right)=s_{j}, j=1, \ldots, k\right)=\prod_{j=1}^{k} \frac{\mu_{j}^{s_{j}}}{s_{j} !} e^{-\mu_{j}} .
$$

For the additional information on Poisson random point processes we refer the reader to [10].

Corollary 1. Let $\lambda_{k}$ be the $k$-th largest eigenvalue of $b_{n}^{-1} A$, then

$$
\lim _{n \rightarrow \infty} \operatorname{Pr}\left(\lambda_{k} \leq x\right)=\exp \left(-x^{-\alpha}\right) \sum_{l=0}^{k-1} \frac{x^{-l \alpha}}{l !} .
$$

In particular, $\lim _{n \rightarrow \infty} \operatorname{Pr}\left(\lambda_{1} \leq x\right)=\exp \left(-x^{-\alpha}\right)$. 
Remark 1 The equivalent formulation of the theorem is the following. Let $k$ be a finite positive integer. Then the joint distribution of the first $k$ largest eigenvalues of $b_{n}^{-1} A$ is asymptotically (in the limit $n \rightarrow \infty$ ) the same as the joint distribution of the first $k$ order statistics of $\left\{b_{n}^{-1}\left|a_{i j}\right|, \quad 1 \leq i \leq j \leq n\right\}$. It is a classical result, that extremal values of the sequence of independent identically distributed random variables with heavy tails distributions (21) have Poisson statistics (see e.g. [23], Theorem 2.3.1).

Theorem 1 was proven in [38] in the Wigner (i.e. full matrix) case (11), (2). The proof in the general (band matrix) case is essentially the same. However, it should be noted, that the original proof of Theorem 1 in 38. contained a little mistake, which could be easily corrected. The corrections are due in two places.

First of all, the correct formulation of the part c) of Lemma 4 from 38 (p. 87 ) should state, that for any positive constant $\delta>0$, with probability going to 1 there is no no row $1 \leq i \leq n$, that contains at least two entries greater in absolute value than $b_{n}^{\frac{3}{4}+\delta}$. In other words, the exponent $\frac{1}{2}+\delta$ in $b_{n}^{\frac{1}{2}+\delta}$ in part c) of Lemma 4 must be replaced by $\frac{3}{4}+\delta$. After this correction, the statement is true. Indeed, the probability that there is a row with at least two entries greater than $b_{n}^{\frac{3}{4}+\delta}$ can be estimated from above by $n^{3}\left(G\left(b_{n}^{\frac{3}{4}+\delta}\right)\right)^{2}$. It follows from (21), (27) and (28), that this probability goes to zero.

Also, the formula (28) in Lemma 5 (p. 88) should read

$$
\operatorname{Pr}\left\{\exists i, 1 \leq i \leq n: \max _{1 \leq j \leq n}\left|a_{i j}\right|>b_{n}^{\frac{3}{4}+\frac{\alpha}{8}}, \quad\left(\sum_{1 \leq j \leq n}\left|a_{i j}\right|\right)-\max _{1 \leq j \leq n}\left|a_{i j}\right|>b_{n}^{\frac{3}{4}+\frac{\alpha}{8}}\right\} \rightarrow 0
$$

as $n \rightarrow \infty$. In other words, the exponent $\frac{1}{2}+\frac{\alpha}{4}$ in $b_{n}^{\frac{1}{2}+\frac{\alpha}{4}}$ must be replaced by $\frac{3}{4}+\frac{\alpha}{8}$. The key step of the proof of Lemma 5 was to show, that for any fixed row $i$ and arbitrary small positive $\delta$, the probability $\operatorname{Pr}\left(\sum_{j:\left|a_{i j}\right| \leq b_{n}^{\frac{1}{2}+\delta}}\left|a_{i j}\right| \geq\right.$ $\left.b_{n}^{\frac{1}{2}+2 \delta}\right)$ can be estimated from above by $\exp \left(-n^{\epsilon}\right)$, where $\epsilon=\epsilon(\delta, \alpha)>0$. We then concluded, that with probability going to 1 , there is no row $i$ such that $\sum_{j:\left|a_{i j}\right| \leq b_{n}^{\frac{1}{2}+\delta}}\left|a_{i j}\right| \geq b_{n}^{\frac{1}{2}+2 \delta}$. To establish (31), it is enough to prove that for any fixed row $i$

$$
\operatorname{Pr}\left(\sum_{j: b_{n}^{1 / 2+\delta} \leq\left|a_{i j}\right| \leq b_{n}^{\frac{3}{4}+\delta}}\left|a_{i j}\right| \geq b_{n}^{\frac{3}{4}+2 \delta}\right)<\exp \left(-n^{\epsilon}\right),
$$

for sufficiently small positive $\epsilon$. The proof is very similar to the argument presented in Lemma 5 , and is left to the reader.

\section{Real Sample Covariance Matrices with Cauchy Entries}

Let $A$ be a rectangular $m \times n$ matrix with independent identically distributed entries with the marginal probability distribution of matrix entries satisfying 
(21). Based on the results in the last section, one can expect that the largest eigenvalues have Poisson statistics as well. At this point, we have been able to prove it only in a weak form, and only when matrix entries have Cauchy distribution.

We recall, that the probability density of the Cauchy distribution is given by the formula $f(x)=\frac{1}{\pi\left(1+x^{2}\right)}$. Cauchy distribution is very important in probability theory (see e.g. [15]). In particular, Cauchy distribition is a $(1,1,0)$ stable distribution, i.e. the scale parameter is 1 , the index of the distribution $\alpha=1$ and the symmetry parameter is zero (see [18, 23]).

The following theorem was proven by Fyodorov and Soshnikov in 39.

Theorem 2. Let $A$ be a random rectangular $m \times n$ matrix $(m \geq n)$ with i.i.d. Cauchy entries and let $z$ be a complex number with a positive real part. Then, as $n \rightarrow \infty$ we have

$$
\lim _{n \rightarrow \infty} E\left(\operatorname{det}\left(1+\frac{z}{m^{2} n^{2}} A^{t} A\right)\right)^{-1 / 2}=\exp \left(-\frac{2}{\pi} \sqrt{z}\right)=\mathbf{E} \prod_{i=1}^{\infty}\left(1+z x_{i}\right)^{-1 / 2},
$$

where we consider the branch of $\sqrt{z}$ on $D=\{z: \Re z>0\}$ such that $\sqrt{1}=1$, $E$ denotes the mathematical expectation with respect to the random matrix ensemble defined above, $\mathbf{E}$ denotes the mathematical expectation with respect to the inhomogeneous Poisson random point process on the positive half-axis with the intensity $\frac{1}{\pi x^{3 / 2}}$, and the convergence is uniform inside $D$ (i.e. it is unform on compact subsets of $D$ ). For a real positive $z=t^{2}, t \in R^{1}$, one can estimate the rate of convergence, namely

$$
\lim _{n \rightarrow \infty} E\left(\operatorname{det}\left(1+\frac{t^{2}}{m^{2} n^{2}} A^{t} A\right)\right)^{-1 / 2}=\exp \left(-\frac{2}{\pi}|t|\left(1+o\left(n^{-1 / 2+\epsilon}\right)\right)\right),
$$

where $\epsilon$ is an arbitrary small positive number and the convergence is uniform on the compact subsets of $[0,+\infty)$.

The result of Theorem 2 allows a generalization to the case of a sparse random matrix with Cauchy entries. Let, as before, $\left\{a_{j k}\right\}, 1 \leq j \leq m, 1 \leq$ $k \leq n$, be i.i.d. Cauchy random variables, and $B=\left(b_{j k}\right)$ be a $m \times n$ nonrandom rectangular $0-1$ matrix such that the number of non-zero entries in each column is fixed and equals to $d_{n}$. Let $d_{n}$ grow polynomially, i.e. $b_{n} \geq n^{\alpha}$, for some $0<\alpha \leq 1$. Also assume that $\ln m$ grows much slower than than any power of $n$.

Define a $m \times n$ rectangular matrix $A$ with the entries $A_{j k}=b_{j k} a_{j k}, \quad 1 \leq$ $j \leq m, 1 \leq k \leq n$. Let $\lambda_{1} \geq \lambda_{2} \ldots \geq \lambda_{n}$ denote the eigenvalues of $A^{t} A$. The appropriate rescaling for the largest eigenvalues in this case is $\tilde{\lambda}_{i}=\frac{\lambda_{i}}{m^{2} d_{n}^{2}}, \quad i=$ $1, \ldots, n$.

Theorem 3. Let $A$ be a sparse random rectangular $m \times n$ matrix $(m \geq n)$ defined as above, and let $z$ be a complex number with a positive real part. Then, as $n \rightarrow \infty$ we have 


$$
\begin{aligned}
& \lim _{n \rightarrow \infty} E\left(\operatorname{det}\left(1+\frac{z}{m^{2} d_{n}^{2}} A^{t} A\right)\right)^{-1 / 2}=\lim _{n \rightarrow \infty} E \prod_{i=1}^{n}\left(1+z \tilde{\lambda}_{i}\right)^{-1 / 2} \\
= & \exp \left(-\frac{2}{\pi} \sqrt{z}\right)=\mathbf{E} \prod_{i=1}^{\infty}\left(1+z x_{i}\right)^{-1 / 2}
\end{aligned}
$$

where, as in Theorem 1.1, we consider the branch of $\sqrt{z}$ on $D=\{z: \Re z>0\}$ such that $\sqrt{1}=1$. $\mathbf{E}$ denotes the mathematical expectation with respect to the inhomogeneous Poisson random point process on the positive half-axis with the intensity $\frac{1}{\pi x^{3 / 2}}$, and the convergence is uniform inside $D$ (i.e. it is unform on the compact subsets of $D)$. For a real positive $z=t^{2}, t \in R^{1}$, one can get an estimate on the rate of convergence, namely

$$
E\left(\operatorname{det}\left(1+\frac{t^{2}}{m^{2} d_{n}^{2}} A^{t} A\right)\right)^{-1 / 2}=\exp \left(-\frac{2}{\pi} t\left(1+o\left(d_{n}^{-1 / 2+\epsilon}\right)\right)\right),
$$

where $\epsilon$ is an arbitrary small positive number and the convergence is uniform on the compact subsets of $[0,+\infty)$.

The proof relies on the following property of the Gaussian integrals:

$$
(\operatorname{det}(B))^{-1 / 2}=\left(\frac{1}{\pi}\right)^{N} \int x \exp \left(-x B x^{t}\right) d^{N},
$$

where $B$ is an $N$-dimensional matrix with a positive definite Hermitian part (i.e. all eigenvalues of $B+B^{*}$ are positive), $x=\left(x_{1}, \ldots, x_{N}\right) \in R^{N}$, and $x B x^{t}=\sum_{i j} b_{i j} x_{i} x_{j}$.

Let $B=B(t)=\left(\begin{array}{cc}I d & t i A \\ t i A^{t} & I d\end{array}\right)$. Then, one can write

$$
\left(\operatorname{det}\left(1+t^{2} A^{t} A\right)\right)^{-1 / 2}=\left(\operatorname{det}\left(\begin{array}{cc}
1 & t i A \\
t i A^{t} & 1
\end{array}\right)\right)^{-1 / 2}=(\operatorname{det}(B))^{-1 / 2}
$$

and apply (38) to the r.h.s. of (39). Assuming that the entries of $A$ are independent, one can significantly simplify the expression, using the fact that entries of $A$ appear linearly in $B(t)$ (see Proposition 1 of [39]). In the Cauchy case, one can simplify the calculations even further, and prove that $\lim _{n \rightarrow \infty} E\left(\operatorname{det}\left(1+\frac{z}{m^{2} d_{n}^{2}} A^{t} A\right)\right)^{-1 / 2}$ exists and equals $\exp \left(-\frac{2}{\pi} \sqrt{z}\right)$.

On the other side, for Poisson random point processes the mathematical expectations of the type $\mathbf{E} \prod_{i=1}^{\infty}\left(1+f\left(x_{i}\right)\right)$ can be calculated explicitely

$$
\begin{aligned}
& \mathbf{E} \prod_{i=1}^{\infty}\left(1+f\left(x_{i}\right)\right)=1+\sum_{k=1}^{\infty} \mathbf{E} \sum_{1 \leq i_{1}<i_{2}<\ldots<i_{k}} \prod_{j=1}^{k} f\left(x_{i_{j}}\right)= \\
= & \sum_{k=0}^{\infty} \frac{1}{k !} \int_{(0,+\infty)^{k}} \prod_{j=1}^{k} f\left(x_{j}\right) \rho_{k}\left(x_{1}, \ldots, x_{k}\right) d x_{1} \cdots d x_{k}
\end{aligned}
$$




$$
=\sum_{k=0}^{\infty} \frac{1}{k !}\left(\int_{(0,+\infty)} f(x) \rho(x) d x\right)^{k}=\exp \left(\int_{(0,+\infty)} f(x) \rho(x) d x\right) .
$$

In the equations above, $\rho_{k}$ denotes the $k$-point correlation function, and $\rho$ denotes the one-point correlation function (also known as intensity). It is a characteristic property of a Poisson random point process that the $\mathrm{k}$-point correlation function factorizes as a product of one-point correlation functions, i.e. $\rho_{k}\left(x_{1}, \ldots, x_{k}\right)=\prod_{i=1}^{k} \rho\left(x_{i}\right)$. In the context of Theorems 2 and 3 , test function $f$ has the form $f(x)=(1+z x)^{-1 / 2}-1$. When the intensity $\rho$ equals $\frac{1}{\pi x^{3 / 2}}$, one obtains

$$
\int_{(0,+\infty)} f(x) \rho(x) d x=\int_{(0,+\infty)}\left((1+z x)^{-1 / 2}-1\right) \frac{1}{\pi x^{3 / 2}} d x=-\frac{2}{\pi} \sqrt{z},
$$

which finishes the proof.

The fact, that the intensity $\rho(x)=\frac{1}{\pi x^{3 / 2}}$ of the Poisson random point process diverges at zero and is summable at $+\infty$, means, that the the vast majority of the eigenvalues of the normalized matrix converge to zero in the limit.

Remark 2 It should be pointed out, that the results of Theorem 2 and 3 do not imply that the statistics of the largest eigenvalues of a normalized sample covariance matrix with Gaussian entries are Poisson in the limit of $n \rightarrow \infty$. Indeed, to prove the Poisson statistics in the limit one has to show that

$$
\lim _{n \rightarrow \infty} E \prod_{i=1}^{n}\left(1+f\left(\tilde{\lambda}_{i}\right)\right)=\mathbf{E} \prod_{i=1}^{+\infty}\left(1+f\left(x_{i}\right)\right)
$$

for a sufficiently large class of the test functions $f$, e.g. for step functions with compact support. As we already pointed out, the results of Theorems 2 and 3 claim that (43) is valid for $f(x)=(1+z x)^{-1 / 2}-1$ for all $z$ such that $\Re z>0$.

\section{Conclusion}

It is known in the theory of random Schrödinger operators, that the statistics of the eigenvalues is Poisson in the localization regime (see e.g. [29], [28]). It seems, that the same mechanism is responsible for the Poisson statistics for the largest eigenvalues in the random matrix models described above. The interesting next problem is to find a phase transition between the TracyWidom regime (when all moments of matrix entries exist) and the Poisson regime (when second moment does not exist).

It is also worth to point out, that there is a vast literature on the Poisson statistics of the energy levels of quantum sysytems in the case of the regular underlying dynamics (see e.g. 44, 35, 33, 9], 25], 26]). 


\section{References}

1. L.Arnold: J. Math. Anal. Appl. 20, 262 (1967)

2. C.W.J. Beenakker: Rev. Mod. Phys., 69, 731, (1997)

3. G. Ben Arous, S.Péché: Commun. Pure Appl. Math., to appear, (2005)

4. M.V.Berry, M. Tabor: Proc. R. Soc. London Ser. A 356, 375 (1977)

5. B.V.Bronk: J. Math. Phys., 6, (1965)

6. A.Casati, L.Molinari, and F.Izrailev: Phys Rev. Lett. 64, 1851 (1990)

7. A.Casati and V.L. Girko: Rand. Oper. Stoch. Equations, 1, 15 (1991)

8. P.Cizeau, J.P.Bouchaud: Phys Rev E, 50, 1810 (1994)

9. Z.Cheng, J.L.Lebowitz and P.Major: Prob. Theo. Rel. Fields, 100, 253 (1994)

10. D.J. Daley, D.Vere-Jones: An Introduction to the Theory of Point Processes, vol.I, 2nd edn, (Springer, Berlin Heidelberg New York 2003)

11. P.Deift Orthogonal Polynomials and Random Matrices: A RiemannHilbert Approach, Courant Lecture Notes in Mathematics, Vol. 3, New York, 1999

12. Z. Füredi and J. Komlós: Combinatorica, 1, 233 (1981)

13. P. Forrester: Nucl. Phys. B, 402, 709 (1994)

14. Y.V.Fyodorov and G. Akemann: JETP Lett. 77, 438 (2003)

15. W. Feller: An Introduction to Probability Theory and Its Applications, Vol. II. 2nd edn. (John Wiley and Sons, Inc., New York, London, Sydney 1971)

16. Y.V. Fyodorov and H.-J.Sommers: J.Phys.A:Math.Gen. 36, 3303 (2003)

17. A.Guionnet: Ann. Inst. H. Poincare Probab. Statist. 38, 341 (2002)

18. I.A.Ibragimov, Yu.V.Linnik, Independent and Stationary Sequences of Random Variables, translation from the Russian edited by J.F.C.Kingman, (WoltersNoordhoff Publishing, Groningen, 1971)

19. A.T.James: Ann. Math. Stat., 35, (1964)

20. K.Johansson: Commun. Math. Phys., 215, 683, (2001)

21. I.M. Johnstone: Ann. Stat., 29, 297 (2001)

22. J. Karamata: Mathematica (Cluj), 4, 38, (1930)

23. M.R. Leadbetter, G.Lindgren and H. Rootzén: Extremes and Related Properties of Random Sequences and Processes, (Springer-Verlag, New York 1983)

24. V.A. Marchenko, L.A. Pastur: Math. USSR-Sb. 1, 457, (1967)

25. J. Marklof: Annals of Mathematics, 158, 419, (2003)

26. J. Marklof: The Berry-Tabor conjecture. In: Proceedings of the 3rd European Congress of Mathematics, Barcelona 2000, (Progress in Mathematics 202 (2001)), pp 421-427

27. M.L.Mehta: Random Matrices, (Academic Press, New York 1991)

28. N.Minami: Commun. Math. Phys., 177, 709, (1996)

29. S.A.Molchanov: Commun. Math. Phys. 78, 429, (1981)

30. S.A. Molchanov, L.A. Pastur and A.M. Khorunzhy: Theor. Math. Phys. 90, 108 (1992)

31. R.J. Muirhead, Aspects of Multivariate Statistical Theory, (Wiley, New York 1982)

32. L.A. Pastur: Teor. Mat. Fiz., 10, 102, (1972)

33. P.Sarnak: Values at integers of binary quadratic forms. In Harmonic Analysis and Number Theory (Montreal, PQ, 1996), CMS Conf. Proc. 21, (Amer. Math. Soc., Providence, RI, 1997), pp 181-203

34. E.Seneta: Regularly Varying Functions, Lecture Notes in Mathematics, 508 (eds. A.Dold and B.Eckmann), (Springer, New York, 1976) 
35. Ya.Sinai: Adv. Sov. Math., AMS Publ., 3, 199, (1991)

36. A.Soshnikov: Commun. Math. Phys., 207, 697, (1999)

37. A.Soshnikov: J. Stat. Phys., 108, 1033, (2002)

38. A.Soshnikov: Elec. Commun. Probab., 9, 82, (2004)

39. A.Soshnikov, Y.Fyodorov: to appear in J. Math Phys. (2005), arXiv preprint math.PR/0403425

40. C.A.Tracy, H.Widom: Commun. Math. Phys., 159, 151, (1994)

41. C.A.Tracy, H.Widom: Commun. Math. Phys., 177, 724, (1996)

42. J.J. Verbaarschot and T. Wettig : Annu.Rev.Nucl.Part.Sci, 50, 343 (2000)

43. S.S.Wilks: Mathematical Statistics, (Princeton University Press, Princeton 1943)

44. E.Wigner: Ann. of Math., 62, 548, (1955)

45. E.Wigner: Ann. of Math., 67, 325, (1958)

46. E.Wigner: SIAM Rev., 9, 1, (1967) 